\title{
Displaying the Structure of the Solutions for Some Fifth-Order Systems of Recursive Equations
}

\author{
H. S. Alayachi, ${ }^{1,2}$ A. Q. Khan $\mathbb{D}^{3}{ }^{3}$ M. S. M. Noorani, ${ }^{1}$ and A. Khaliq ${ }^{4}$ \\ ${ }^{1}$ School of Mathematical Sciences, Faculty of Science and Technology, Universiti Kebangsaan Malaysia, Bangi, Selangor, Malaysia \\ ${ }^{2}$ Department of Mathematics, College of Science, Taibah University, Al-Madinah Al-Munawarah, Saudi Arabia \\ ${ }^{3}$ Department of Mathematics, University of Azad Jammu and Kashmir, Muzaffarabad 13100, Pakistan \\ ${ }^{4}$ Department of Mathematics, Riphah Institute of Computing and Applied Sciences, Riphah International University, \\ Lahore Campus, Lahore, Pakistan
}

Correspondence should be addressed to A. Q. Khan; abdulqadeerkhan1@gmail.com

Received 9 November 2020; Revised 18 December 2020; Accepted 30 December 2020; Published 12 January 2021

Academic Editor: Cemil Tunç

Copyright (C) 2021 H. S. Alayachi et al. This is an open access article distributed under the Creative Commons Attribution License, which permits unrestricted use, distribution, and reproduction in any medium, provided the original work is properly cited.

This paper presents the solutions to the following nonlinear systems of rational difference equations: $x_{n+1}=\left(\left(x_{n-3} y_{n-4}\right) /\left(y_{n}(1+\right.\right.$ $\left.\left.\left.x_{n-1} y_{n-2} x_{n-3} y_{n-4}\right)\right)\right), y_{n+1}=\left(\left(y_{n-3} x_{n-4}\right) /\left(x_{n}\left( \pm 1 \pm y_{n-1} x_{n-2} y_{n-3} x_{n-4}\right)\right)\right)$ where initial conditions $x_{-\delta}, y_{-\delta}(\delta=4,3, \ldots, 0)$ are nonnegative real numbers. Finally some numerical simulations are presented to verify obtained theoretical results.

\section{Introduction}

The purpose of present study is to solve and deal with the following difference equations systems:

$$
\begin{aligned}
& x_{n+1}=\frac{x_{n-3} y_{n-4}}{y_{n}\left(1+x_{n-1} y_{n-2} x_{n-3} y_{n-4}\right)}, \\
& y_{n+1}=\frac{y_{n-3} x_{n-4}}{x_{n}\left( \pm 1 \pm y_{n-1} x_{n-2} y_{n-3} x_{n-4}\right)},
\end{aligned}
$$

where $x_{-\delta}, y_{-\delta}(\delta=4,3, \ldots, 0)$ are arbitrary nonnegative real numbers.

It is anticipated that discrete dynamical systems can be seen as discrete analogous of differential as well as delay differential equations. Moreover, these systems designate certain natural phenomena in economy, physics, biology, and many more. Many scholars and researchers have studied various dynamical properties of difference equations along their systems in recent years. For example, Asiri et al. [1] have investigated the periodicity nature of the following system:

$$
\begin{aligned}
& x_{n+1}=\frac{y_{n-2}}{-y_{n-2} x_{n-1} y_{n}+1}, \\
& y_{n+1}=\frac{x_{n-2}}{ \pm x_{n-2} y_{n-1} x_{n} \pm 1} .
\end{aligned}
$$

Cinar et al. [2] have obtained the solution of the subsequent recursive system:

$$
\begin{aligned}
& x_{n+1}=\frac{x_{n-1}}{y_{n} x_{n-1}-1}, \\
& y_{n+1}=\frac{y_{n-1}}{x_{n} y_{n-1}-1}, \\
& z_{n+1}=\frac{x_{n}}{y_{n} z_{n-1}} .
\end{aligned}
$$

Metwally and Elsayed [3] have explored the periodicity nature of the following system:

$$
\begin{aligned}
& x_{n+1}=\frac{y_{n-2}}{-y_{n-2} x_{n-1} y_{n}-1}, \\
& y_{n+1}=\frac{x_{n-2}}{ \pm x_{n-2} y_{n-1} x_{n} \pm 1} .
\end{aligned}
$$


Touafek et al. [4] have explored the periodicity nature of the following system:

$$
\begin{aligned}
& x_{n+1}=\frac{x_{n-3}}{ \pm x_{n-3} y_{n-1} \pm 1}, \\
& y_{n+1}=\frac{y_{n-3}}{ \pm y_{n-3} x_{n-1} \pm 1} .
\end{aligned}
$$

Elsayed [5] has obtained the solution of the following rational system:

$$
\begin{aligned}
& x_{n+1}=\frac{x_{n-1}}{ \pm x_{n-1} y_{n} \pm 1} \\
& y_{n+1}=\frac{y_{n-1}}{y_{n-1} x_{n} \mp 1} .
\end{aligned}
$$

For more interesting results regarding dynamical properties of difference and differential equations along their systems, we refer the readers to [2,6-28] and the references cited therein.

\section{On $1^{\text {st }}$ System}

$$
\begin{aligned}
& x_{n+1}=\frac{x_{n-3} y_{n-4}}{y_{n}\left(1+x_{n-1} y_{n-2} x_{n-3} y_{n-4}\right)}, \\
& y_{n+1}=\frac{y_{n-3} x_{n-4}}{x_{n}\left(1+y_{n-1} x_{n-2} y_{n-3} x_{n-4}\right)} .
\end{aligned}
$$

This section is about the investigation of the solution form of the following system:

$$
\begin{aligned}
& x_{n+1}=\frac{x_{n-3} y_{n-4}}{y_{n}\left(1+x_{n-1} y_{n-2} x_{n-3} y_{n-4}\right)}, \\
& y_{n+1}=\frac{y_{n-3} x_{n-4}}{x_{n}\left(1+y_{n-1} x_{n-2} y_{n-3} x_{n-4}\right)} .
\end{aligned}
$$

The forms of solutions to (8) are given as Theorem 1 .

Theorem 1. Let $\left\{x_{n}, y_{n}\right\}$ be a solution to (8), and also let $x_{-\delta}, y_{-\delta}(\delta=4,3, \ldots, 0)$, respectively, be $a, b, c, d, e, f$, $g, h, k, l$. Then one has

$$
\begin{aligned}
& x_{4 n-4}=\frac{e^{n} \prod_{\delta=0}^{-1+n}[2 \delta a c g k+1]}{a^{n-1} \prod_{\delta=0}^{-1+n}[2 \delta c e g k+1]}, \\
& x_{4 n-3}=\frac{b f^{n} \prod_{\delta=0}^{-1+n}[2 \delta b d h l+1]}{l^{n} \prod_{\delta=0}^{-1+n}[(2 \delta+1) b d f h+1]}, \\
& x_{4 n-2}=\frac{c e^{n} \prod_{\delta=0}^{-1+n}[(1+2 \delta) a c g k+1]}{a^{n} \prod_{\delta=0}^{-1+n}[(1+2 \delta) c e g k+1]}, \\
& x_{4 n-1}=\frac{d f^{n} \prod_{i=1}^{-1+n}[(1+2 \delta) b d h l+1]}{l^{n} \prod_{\delta=0}^{-1+n}[(2+2 \delta) b d f h+1]},
\end{aligned}
$$

$$
\begin{aligned}
& y_{4 n-4}=\frac{l^{n} \prod_{\delta=0}^{-1+n}[2 \delta b d f h+1]}{f^{-1+n} \prod_{\delta=0}^{-1+n}[2 \delta b d h l+1]}, \\
& y_{4 n-3}=\frac{g a^{n} \prod_{\delta=0}^{-1+n}[2 \delta c e g k+1]}{e^{n} \prod_{\delta=0}^{-1+n}[(1+2 \delta) a c g k+1]}, \\
& y_{4 n-2}=\frac{h l^{n} \prod_{\delta=0}^{-1+n}[(2 \delta+1) b d f h+1]}{f^{n} \prod_{\delta=0}^{-1+n}[(1+2 \delta) b d h l+1]}, \\
& y_{4 n-1}=\frac{k a^{n} \prod_{\delta=0}^{-1+n}[(2 \delta+1) c e g k+1]}{e^{n} \prod_{\delta=0}^{-1+n}[(2 \delta+2) a c g k+1]} .
\end{aligned}
$$

Proof. Obviously results true if $n=0$. Assuming that for $n-$ 1 and $n-2$ results hold, that is

$$
\begin{aligned}
& x_{4 n-9}=\frac{d f^{-2+n} \prod_{\delta=0}^{n-3}[(2 \delta+1) b d h l+1]}{l^{n-2} \prod_{\delta=0}^{n-3}[(2+2 \delta) b d f h+1]}, \\
& x_{4 n-8}=\frac{e^{n-1} \prod_{\delta=0}^{-2+n}[2 \delta \text { acgki }+1]}{a^{n-2} \prod_{\delta=0}^{-2+n}[2 \delta \text { cegk }+1]}, \\
& x_{4 n-7}=\frac{b f^{-1+n} \prod_{\delta=0}^{-2+n}[2 \delta b d h l+1]}{l^{n-1} \prod_{\delta=0}^{-2+n}[(1+2 \delta) b d f h+1]}, \\
& x_{4 n-6}=\frac{c e^{n-1} \prod_{\delta=0}^{-2+n}[(2 \delta+1) \operatorname{acgk}+1]}{a^{n-1} \prod_{\delta=0}^{-2+n}[(2 \delta+1) \operatorname{ceg} k+1]}, \\
& x_{4 n-5}=\frac{d \times f^{-1+n} \prod_{\delta=0}^{-2+n}[(2 \delta+1) b d h l+1]}{l^{n-1} \prod_{\delta=0}^{-2+n}[(2+2 \delta) b d f h+1]}, \\
& y_{4 n-9}=\frac{k a^{n-2} \prod_{\delta=0}^{n-3}[(2 \delta+1) \operatorname{ceg} k+1]}{e^{n-2} \prod_{\delta=0}^{n-3}[(2+2 \delta) a c g k+1]}, \\
& y_{4 n-8}=\frac{l^{n-1} \prod_{\delta=0}^{-2+n}[2 \delta b d f h+1]}{f^{-2+n} \prod_{\delta=0}^{-2+n}[2 \delta b d h l+1]}, \\
& y_{4 n-7}=\frac{g a^{n-1} \prod_{\delta=0}^{-2+n}[2 \delta c e g k+1]}{e^{n-1} \prod_{\delta=0}^{-2+n}[(2 \delta+1) a c g k+1]}, \\
& y_{4 n-6}=\frac{h l^{n-1} \prod_{\delta=0}^{-2+n}[(2 \delta+1) b d f h+1]}{f^{-1+n} \prod_{\delta=0}^{-2+n}[(1+2 \delta) b d h l+1]}, \\
& y_{4 n-5}=\frac{k a^{n-1} \prod_{\delta=0}^{-2+n}[(2 \delta+1) \operatorname{ceg} k+1]}{e^{n-1} \prod_{\delta=0}^{-2+n}[(2+2 \delta) a c g k+1]} .
\end{aligned}
$$

Now from (8), one has 


$$
\begin{aligned}
& x_{4 n-4}=\frac{x_{4 n-8} y_{4 n-9}}{y_{4 n-5}\left(1+x_{4 n-6} y_{4 n-7} x_{4 n-8} y_{4 n-9}\right)} \\
& =\frac{\left(\left(e^{n-1} \prod_{\delta=0}^{-2+n}[2 \delta a c g k+1]\right) /\left(a^{n-2} \prod_{\delta=0}^{-2+n}[2 \delta c e g k+1]\right)\right)\left(\left(k a^{n-2} \prod_{\delta=0}^{n-3}[(1+2 \delta) \operatorname{ceg} k+1]\right) /\left(e^{n-2} \prod_{\delta=0}^{n-3}[(2 \delta+2) a c g k+1]\right)\right)}{\left(\left(k a^{n-1} \prod_{\delta=0}^{-2+n}[(2 \delta+1) \operatorname{ceg} k+1]\right) /\left(e^{n-1} \prod_{\delta=0}^{-2+n}[(2 \delta+2) \operatorname{acg} k+1]\right)\right)} \\
& \left(1+\left(\left(c e^{n-1} \prod_{\delta=0}^{-2+n}[(2 \delta+1) a c g k+1]\right) /\left(a^{n-1} \prod_{\delta=0}^{-2+n}[(2 \delta+1) c e g k+1]\right)\right)\right. \\
& \left(\left(g a^{n-1} \prod_{\delta=0}^{n-2}[2 \delta c e g k+1]\right) /\left(e^{n-1} \prod_{\delta=0}^{n-2}[(2 \delta+1) a c g k+1]\right)\right) \\
& \left(\left(e^{n-1} \prod_{\delta=0}^{-2+n}[2 \delta a c g k+1]\right) /\left(a^{n-2} \prod_{\delta=0}^{-2+n}[(2 \delta) c e g k+1]\right)\right) \\
& \left.\left(\left(k a^{n-2} \prod_{\delta=0}^{n-3}[(2 \delta+1) c e g k+1]\right) /\left(e^{n-2} \prod_{\delta=0}^{n-3}[(2 \delta+2) a c g k+1]\right)\right)\right) \\
& \left(\left(e k \prod_{\delta=0}^{n-3}[(2 \delta+1) c e g k+1]\right) /\left(\prod_{\delta=0}^{-2+n}[(2 \delta) c e g k+1]\right)\right)
\end{aligned}
$$

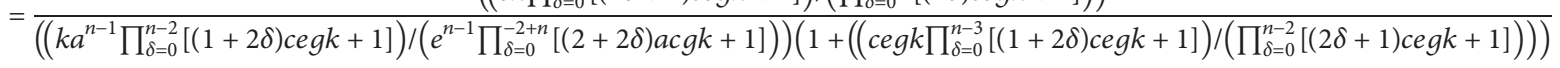

$$
\begin{aligned}
& e^{n} \prod_{\delta=0}^{-2+n}[(2+2 \delta) a c g k+1]
\end{aligned}
$$

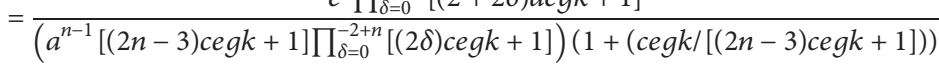

$$
\begin{aligned}
& =\frac{e^{n} \prod_{\delta=0}^{-2+n}[(2 \delta+2) \operatorname{acg} k+1]}{\left(a^{n-1} \prod_{\delta=0}^{-2+n}[(2 \delta) \operatorname{ceg} k+1]\right)([(2 n-3) \operatorname{ceg} k+1]+\operatorname{ceg} k)} \\
& =\frac{e^{n} \prod_{\delta=0}^{-2+n}[(2+2 \delta) \operatorname{acg} k+1]}{\left(a^{n-1} \prod_{\delta=0}^{-2+n}[(2 \delta) \operatorname{ceg} k+1]\right)[(-2+2 n) \operatorname{ceg} k+1]} \\
& =\frac{e^{n} \prod_{\delta=0}^{-1+n}[(2 \delta) \operatorname{acg} k+1]}{a^{n-1} \prod_{\delta=0}^{-1+n}[(2 \delta) \operatorname{ceg} k+1]} \\
& y_{4 n-4}=\frac{y_{4 n-8} x_{4 n-9}}{x_{4 n-5}\left(1+y_{4 n-6} x_{4 n-7} y_{4 n-8} x_{4 n-9}\right)} \\
& =\frac{\left(\left(l^{n-1} \prod_{\delta=0}^{-2+n}[2 \delta b d f h+1]\right) /\left(f^{-2+n} \prod_{\delta=0}^{-2+n}[2 \delta b d h l+1]\right)\right)\left(\left(d f^{-2+n} \prod_{\delta=0}^{n-3}[(1+2 \delta) b d h l+1]\right) /\left(l^{n-2} \prod_{\delta=0}^{n-3}[(2+2 \delta) b d f h+1]\right)\right)}{\left(\left(d f^{-1+n} \prod_{\delta=0}^{-2+n}[(1+2 \delta) b d h l+1]\right) /\left(l^{n-1} \prod_{\delta=0}^{-2+n}[(2+2 \delta) b d f h+1]\right)\right)} \\
& \left(1+\left(\left(h l^{n-1} \prod_{\delta=0}^{-2+n}[(2 \delta+1) b d f h+1]\right) /\left(f^{-1+n} \prod_{\delta=0}^{-2+n}[(1+2 \delta) b d h l+1]\right)\right)\right. \\
& \left(\left(b f^{-1+n} \prod_{\delta=0}^{n-2}[(2 \delta) b d h l+1]\right) /\left(l^{n-1} \prod_{\delta=0}^{n-2}[(1+2 \delta) b d f h+1]\right)\right) \\
& \left(\left(l^{n-1} \prod_{\delta=0}^{-2+n}[(2 \delta) b d f h+1]\right) /\left(f^{-2+n} \prod_{\delta=0}^{-2+n}[(2 \delta) b d h l+1]\right)\right) \\
& \left.\left(\left(d f^{-2+n} \prod_{\delta=0}^{n-3}[(1+2 \delta) b d h l+1]\right) / l^{n-2} \prod_{\delta=0}^{n-3}[(2+2 \delta) b d f h+1]\right)\right) \\
& =\frac{\left(\left(l^{n-1} \prod_{\delta=0}^{-2+n}[(2 \delta) b d f h+1]\right) /\left(f^{-2+n} \prod_{\delta=0}^{-2+n}[2 \delta b d h l+1]\right)\right)\left(\left(d f^{-2+n} \prod_{\delta=0}^{n-3}[(2 \delta+1) b d h l+1]\right) /\left(l^{n-2} \prod_{\delta=0}^{n-3}[(2 \delta+2) b d f h+1]\right)\right)}{\left(\left(d f^{-1+n} \prod_{\delta=0}^{-2+n}[(2 \delta+1) b d h l+1]\right) /\left(l^{n-1} \prod_{\delta=0}^{-2+n}[(2 \delta+2) b d f h+1]\right)\right)\left(1+\left(\left(b d h l \prod_{\delta=0}^{n-3}[(2 \delta+1) b d h l+1]\right) /\left(\prod_{\delta=0}^{n-2}[(2 \delta+1) b d h l+1]\right)\right)\right)} \\
& =\frac{l^{n} \prod_{\delta=0}^{-2+n}[(2 \delta+2) b d f h+1]}{f^{-1+n}[(2 n-3) b d h l+1] \prod_{\delta=0}^{-2+n}[2 \delta b d h l+1](1+(b d h l /[(2 n-3) b d h l+1]))} \\
& =\frac{l^{n} \prod_{\delta=0}^{-2+n}[(2+2 \delta) b d f h+1]}{f^{-1+n} \prod_{\delta=0}^{-2+n}[2 \delta b d h l+1]([(2 n-3) b d h l+1]+b d h l)} \\
& =\frac{l^{n} \prod_{\delta=0}^{-2+n}[(2 \delta+2) b d f h+1]}{f^{-1+n} \prod_{\delta=0}^{-2+n}[(2 \delta) b d h l+1][(2 n-2) b d h l]} \\
& =\frac{l^{n} \prod_{\delta=0}^{-1+n}[(2 \delta) b d f h+1]}{f^{-1+n} \prod_{\delta=0}^{-1+n}[(2 \delta) b d h l+1]} .
\end{aligned}
$$


Again, from system (8), we obtain

$$
\begin{aligned}
& x_{4 n-3}=\frac{x_{4 n-7} y_{4 n-8}}{y_{4 n-4}\left(1+x_{4 n-5} y_{4 n-6} x_{4 n-7} y_{4 n-8}\right)} \\
& =\frac{\left(\left(b f^{-1+n} \prod_{\delta=0}^{-2+n}[(2 \delta) b d h l+1]\right) /\left(l^{n-1} \prod_{\delta=0}^{-2+n}[(2 \delta+1) b d f h+1]\right)\right)\left(\left(l^{n-1} \prod_{\delta=0}^{n-2}[(2 \delta) b d f h+1]\right) /\left(f^{-2+n} \prod_{\delta=0}^{n-2}[(2 \delta) b d h l+1]\right)\right)}{\left(\left(l^{n} \prod_{\delta=0}^{-1+n}[(2 \delta) b d f h+1]\right) /\left(f^{-1+n} \prod_{\delta=0}^{-1+n}[(2 \delta) b d h l+1]\right)\right)} \\
& \left(1+\left(\left(d f^{-1+n} \prod_{\delta=0}^{-2+n}[(2 \delta+1) b d h l+1]\right) /\left(l^{n-1} \prod_{\delta=0}^{-2+n}[(2 \delta+2) b d f h+1]\right)\right)\right. \\
& \left(\left(h l^{n-1} \prod_{\delta=0}^{n-2}[(2 \delta+1) b d f h+1]\right) /\left(f^{-1+n} \prod_{\delta=0}^{n-2}[(2 \delta+1) b d h l+1]\right)\right) \\
& \left(\left(b f^{-1+n} \prod_{\delta=0}^{-2+n}[(2 \delta) b d h l+1]\right) /\left(l^{n-1} \prod_{\delta=0}^{-2+n}[(2 \delta+1) b d f h+1]\right)\right) \\
& \left.\left(\left(l^{n-1} \prod_{\delta=0}^{n-2}[(2 \delta) b d f h+1]\right) /\left(f^{-2+n} \prod_{\delta=0}^{n-2}[(2 \delta) b d h l+1]\right)\right)\right) \\
& =\frac{b f^{n} \prod_{\delta=0}^{-2+n}[(2 \delta) b d f h+1] \prod_{\delta=0}^{-1+n}[(2 \delta) b d h l+1]}{l^{n} \prod_{\delta=0}^{-1+n}[(2 \delta) b d f h+1] \prod_{\delta=0}^{-2+n}[(2 \delta+1) b d f h+1]\left(1+\left(\left(b d f h \prod_{\delta=0}^{n-2}[(2 \delta) b d f h+1]\right) /\left(\prod_{\delta=0}^{n-2}[(2 \delta+2) b d f h+1]\right)\right)\right)} \\
& =\frac{b f^{n} \prod_{\delta=0}^{-1+n}[(2 \delta) b d h l+1]}{l^{n}[(2 n-2) b d f h+1] \prod_{\delta=0}^{-2+n}[(2 \delta+1) b d f h+1](1+(b d f h /[(2 n-2) b d f h+1]))} \\
& =\frac{b f^{n} \prod_{\delta=0}^{-1+n}[(2 \delta) b d h l+1]}{l^{n} \prod_{\delta=0}^{-2+n}[(2 \delta+1) b d f h+1]([(2 n-2) b d f h+1]+b d f h)} \\
& =\frac{b f^{n} \prod_{\delta=0}^{-1+n}[(2 \delta) b d h l+1]}{l^{n} \prod_{\delta=0}^{-2+n}[(2 \delta+1) b d f h+1][(-1+2 n) b d f h+1]} \\
& =\frac{b f^{n} \prod_{\delta=0}^{-1+n}[(2 \delta) b d h l+1]}{l^{n} \prod_{\delta=0}^{-1+n}[(2 \delta+1) b d f h+1]} \\
& y_{4 n-3}=\frac{y_{4 n-7} x_{4 n-8}}{x_{4 n-4}\left(1+y_{4 n-5} x_{4 n-6} y_{4 n-7} x_{4 n-8}\right)} \\
& =\frac{\left(\left(g a^{n-1} \prod_{\delta=0}^{-2+n}[(2 \delta) c e g k+1]\right) /\left(e^{n-1} \prod_{\delta=0}^{-2+n}[(2 \delta+1) a c g k+1]\right)\right)\left(\left(e^{n-1} \prod_{\delta=0}^{n-2}[(2 \delta) a c g k+1]\right) /\left(a^{n-2} \prod_{\delta=0}^{n-2}[(2 \delta) c e g k+1]\right)\right)}{\left(\left(e^{n} \prod_{\delta=0}^{-1+n}[(2 \delta) a c g k+1]\right) /\left(a^{n-1} \prod_{\delta=0}^{-1+n}[(2 \delta) c e g k+1]\right)\right)} \\
& \left(1+\left(\left(k a^{n-1} \prod_{\delta=0}^{-2+n}[(1+2 \delta) c e g k+1]\right) /\left(e^{n-1} \prod_{\delta=0}^{-2+n}[(2 \delta+2) a c g k+1]\right)\right)\right. \\
& \left(\left(c e^{n-1} \prod_{\delta=0}^{n-2}[(1+2 \delta) a c g k+1]\right) / a^{n-1} \prod_{\delta=0}^{n-2}[(2 \delta+1) c e g k+1]\right) \\
& \left(\left(g a^{n-1} \prod_{\delta=0}^{-2+n}[(2 \delta) c e g k+1]\right) /\left(e^{n-1} \prod_{\delta=0}^{-2+n}[(2 \delta+1) a c g k+1]\right)\right) \\
& \left.\left(\left(e^{n-1} \prod_{\delta=0}^{n-2}[(2 \delta) a c g k+1]\right) /\left(a^{n-2} \prod_{\delta=0}^{n-2}[(2 \delta) c e g k+1]\right)\right)\right) \\
& =\frac{g a^{n} \prod_{\delta=0}^{-1+n}[(2 \delta) \operatorname{ceg} k+1]}{e^{n} \prod_{\delta=0}^{-2+n}[(2 \delta+1) \operatorname{acgk}+1][(2 n-2) a c g k+1](1+(\operatorname{acgk} /[(2 n-2) \operatorname{acgk}+1]))} \\
& =\frac{g a^{n} \prod_{\delta=0}^{-1+n}[(2 \delta) \operatorname{ceg} k+1]}{e^{n} \prod_{\delta=0}^{-2+n}[(2 \delta+1) a c g k+1]([(2 n-2) a c g k+1]+a c g k)} \\
& =\frac{g a^{n} \prod_{\delta=0}^{-1+n}[(2 \delta) c e g k+1]}{e^{n} \prod_{\delta=0}^{-2+n}[(2 \delta+1) a c g k+1][(-1+2 n) a c g k+1]} \\
& =\frac{g a^{n} \prod_{\delta=0}^{-1+n}[(2 \delta) c e g k+1]}{e^{n} \prod_{\delta=0}^{-1+n}[(1+2 \delta) a c g k+1]} .
\end{aligned}
$$


Hence, the proof of other relations is obvious.

\section{On $2^{\text {nd }}$ System}

$$
\begin{aligned}
& x_{n+1}=\frac{x_{n-3} y_{n-4}}{y_{n}\left(1+x_{n-1} y_{n-2} x_{n-3} y_{n-4}\right)}, \\
& y_{n+1}=\frac{y_{n-3} x_{n-4}}{x_{n}\left(1-y_{n-1} x_{n-2} y_{n-3} x_{n-4}\right)} .
\end{aligned}
$$

In this section, we explore the solutions of the recursive system:

$$
\begin{aligned}
& x_{n+1}=\frac{x_{n-3} y_{n-4}}{y_{n}\left(1+x_{n-1} y_{n-2} x_{n-3} y_{n-4}\right)}, \\
& y_{n+1}=\frac{y_{n-3} x_{n-4}}{x_{n}\left(1-y_{n-1} x_{n-2} y_{n-3} x_{n-4}\right)} .
\end{aligned}
$$

Theorem 2. Let $\left\{x_{n}, y_{n}\right\}$ be a solution to (14), and also let $x_{-\delta}, y_{-\delta}(\delta=4,3, \ldots, 0)$, respectively, be $a, b, c, d, e, f, g, h$, $k$, l; then

$$
\begin{aligned}
& x_{4 n-4}=\frac{e^{n} \prod_{\delta=0}^{-1+n}[1-(2 \delta) a c g k]}{a^{n-1} \prod_{\delta=0}^{-1+n}[1+(2 \delta) c e g k]}, \\
& x_{4 n-3}=\frac{b f^{n} \prod_{\delta=0}^{-1+n}[1-(2 \delta) b d h l]}{l^{n} \prod_{\delta=0}^{-1+n}[1+(2 \delta+1) b d f h]}, \\
& x_{4 n-2}=\frac{c e^{n} \prod_{i=1}^{n-1}[1-(2 \delta+1) a c g k]}{a^{n} \prod_{\delta=0}^{-1+n}[1+(1+2 \delta) c e g k]}, \\
& x_{4 n-1}=\frac{d f^{n} \prod_{\delta=0}^{-1+n}[1-(2 \delta+1) b d h l]}{l^{n} \prod_{\delta=0}^{-1+n}[1+(2 \delta+2) b d f h]}, \\
& y_{4 n-4}=\frac{l^{n} \prod_{\delta=0}^{-1+n}[1+(2 \delta) b d f h]}{f^{-1+n} \prod_{\delta=0}^{-1+n}[1-(2 \delta) b d h l]}, \\
& y_{4 n-3}=\frac{g a^{n} \prod_{\delta=0}^{-1+n}[1+(2 \delta) c e g k]}{e^{n} \prod_{\delta=0}^{-1+n}[1-(2 \delta+1) a c g k]}, \\
& y_{4 n-2}=\frac{h l^{n} \prod_{\delta=0}^{-1+n}[1+(2 \delta+1) b d f h]}{f^{n} \prod_{\delta=0}^{-1+n}[1-(2 \delta+1) b d h l]}, \\
& y_{4 n-1}=\frac{k a^{n} \prod_{\delta=0}^{-1+n}[1+(2 \delta+1) c e g k]}{e^{n} \prod_{\delta=0}^{-1+n}[1-(2 \delta+2) a c g k]} .
\end{aligned}
$$

Proof. Obviously results true if $n=0$. Assuming that for $n-$ 1 and $n-2$ results hold, that is

$$
\begin{aligned}
& x_{4 n-9}=\frac{d f^{-2+n} \prod_{\delta=0}^{n-3}[1-(2 \delta+1) b d h l]}{l^{n-2} \prod_{\delta=0}^{n-3}[1+(2 \delta+2) b d f h]}, \\
& x_{4 n-8}=\frac{e^{n-1} \prod_{\delta=0}^{-2+n}[1-(2 \delta) \operatorname{acgk}]}{a^{n-2} \prod_{\delta=0}^{-2+n}[1+(2 \delta) \text { cegk }]}, \\
& x_{4 n-7}=\frac{b f^{-1+n} \prod_{\delta=0}^{-2+n}[1-(2 \delta) b d h l]}{l^{n-1} \prod_{\delta=0}^{-2+n}[1+(2 \delta+1) b d f h]}, \\
& x_{4 n-6}=\frac{c e^{n-1} \prod_{i=1}^{n-2}[1-(2 \delta+1) a c g k]}{a^{n-1} \prod_{\delta=0}^{2+n}[1+(1+2 \delta) c e g k]}, \\
& x_{4 n-5}=\frac{d f^{-1+n} \prod_{\delta=0}^{-2+n}[1-(2 \delta+1) b d h l]}{l^{n-1} \prod_{\delta=0}^{-2+n}[1+(2+2 \delta) b d f h]}, \\
& y_{4 n-9}=\frac{k a^{n-2} \prod_{\delta=0}^{n-3}[1+(2 \delta+1) c e g k]}{e^{n-2} \prod_{\delta=0}^{n-3}[1-(2+2 \delta) a c g k]}, \\
& y_{4 n-8}=\frac{l^{n-1} \prod_{\delta=0}^{-2+n}[1+(2 \delta) b d f h]}{f^{-2+n} \prod_{\delta=0}^{-2+n}[1-(2 \delta) b d h l]}, \\
& y_{4 n-7}=\frac{g a^{n-1} \prod_{\delta=0}^{-2+n}[1+(2 \delta) \text { cegk }]}{e^{n-1} \prod_{\delta=0}^{-2+n}[1-(2 \delta+1) \operatorname{acg} k]}, \\
& y_{4 n-6}=\frac{h l^{n-1} \prod_{\delta=0}^{-2+n}[1+(2 \delta+1) b d f h]}{f^{-1+n} \prod_{\delta=0}^{-2+n}[1-(2 \delta+1) b d h l]}, \\
& y_{4 n-5}=\frac{k a^{n-1} \prod_{\delta=0}^{-2+n}[1+(2 \delta+1) \operatorname{ceg} k]}{e^{n-1} \prod_{\delta=0}^{-2+n}[1-(2 \delta+2) a c g k]} \text {. }
\end{aligned}
$$

Next, one can obtain from system (14) that 


$$
\begin{aligned}
& x_{4 n-4}=\frac{x_{4 n-8} y_{4 n-9}}{y_{4 n-5}\left(1+x_{4 n-6} y_{4 n-7} x_{4 n-8} y_{4 n-9}\right)} \\
& =\frac{\left(\left(e^{n-1} \prod_{\delta=0}^{-2+n}[1-(2 \delta) a c g k]\right) /\left(a^{n-2} \prod_{\delta=0}^{-2+n}[1+(2 \delta) c e g k]\right)\right)\left(\left(k a^{n-2} \prod_{\delta=0}^{n-3}[1+(1+2 \delta) c e g k]\right) /\left(e^{n-2} \prod_{\delta=0}^{n-3}[1-(2+2 \delta) a c g k]\right)\right)}{\left(\left(k a^{n-1} \prod_{\delta=0}^{-2+n}[1+(2 \delta+1) c e g k]\right) /\left(e^{n-1} \prod_{\delta=0}^{-2+n}[1-(2 \delta+2) a c g k]\right)\right)} \\
& \left(1+\left(\left(c e^{n-1} \prod_{i=1}^{n-2}[1-(2 \delta+1) a c g k]\right) /\left(a^{n-1} \prod_{\delta=0}^{-2+n}[1+(2 \delta+1) \operatorname{ceg} k]\right)\right)\right. \\
& \left(\left(g a^{n-1} \prod_{\delta=0}^{n-2}[1+(2 \delta) c e g k]\right) /\left(e^{n-1} \prod_{\delta=0}^{-2+n}[1-(2 \delta+1) a c g k]\right)\right) \\
& \left(\left(e^{n-1} \prod_{\delta=0}^{-2+n}[1-(2 \delta) \operatorname{acg} k]\right) /\left(a^{n-2} \prod_{\delta=0}^{-2+n}[1+(2 \delta) \operatorname{ceg} k]\right)\right) \\
& \left.\left(\left(k a^{n-2} \prod_{\delta=0}^{n-3}[1+(2 \delta+1) c e g k]\right) /\left(e^{n-2} \prod_{\delta=0}^{n-3}[1-(2 \delta+2) a c g k]\right)\right)\right) \\
& =\frac{e^{n} \prod_{\delta=0}^{n-3}[1+(1+2 \delta) \operatorname{ceg} k] \prod_{\delta=0}^{-2+n}[1-(2+2 \delta) \operatorname{acg} k]}{\left(a^{n-1} \prod_{\delta=0}^{-2+n}[1+(2 \delta+1) \operatorname{ceg} k] \prod_{\delta=0}^{-2+n}[1+(2 \delta) \operatorname{ceg} k]\right)(1+(\operatorname{ceg} k /[1+(2 n-3) \operatorname{ceg} k]))} \\
& =\frac{e^{n} \prod_{\delta=0}^{-2+n}[1-(2+2 \delta) \operatorname{acgk}]}{\left(a^{n-1}[1+(2 n-3) \operatorname{ceg} k] \prod_{\delta=0}^{-2+n}[1+(2 \delta) \operatorname{cegk}]\right)(1+(\text { cegk } /[1+(2 n-3) \operatorname{ceg} k]))} \\
& =\frac{e^{n} \prod_{\delta=0}^{-2+n}[1-(2 \delta+2) a c g k]}{\left(a^{n-1} \prod_{\delta=0}^{-2+n}[1+(2 \delta) c e g k]\right)([1+(2 n-3) c e g k]+c e g k)} \\
& =\frac{e^{n} \prod_{\delta=0}^{-2+n}[1-(2+2 \delta) a c g k]}{\left(a^{n-1} \prod_{\delta=0}^{-2+n}[1+(2 \delta) c e g k]\right)(1+(2 n-2) c e g k)} \\
& =\frac{e^{n} \prod_{\delta=0}^{-1+n}[1-(2 \delta) a c g k]}{a^{n-1} \prod_{\delta=0}^{-1+n}[1+(2 \delta) c e g k]} \text {. }
\end{aligned}
$$

Similarly, system (14) gives

$$
\begin{aligned}
& y_{4 n-4}=\frac{y_{4 n-8} x_{4 n-9}}{x_{4 n-5}\left(1-y_{4 n-6} x_{4 n-7} y_{4 n-8} x_{4 n-9}\right)} \\
& =\frac{\left(\left(l^{n-1} \prod_{\delta=0}^{-2+n}[1+(2 \delta) b d f h]\right) /\left(f^{-2+n} \prod_{\delta=0}^{-2+n}[1-(2 \delta) b d h l]\right)\right)\left(\left(d f^{-2+n} \prod_{\delta=0}^{n-3}[1-(2 \delta+1) b d h l]\right) /\left(l^{n-2} \prod_{\delta=0}^{n-3}[1+(2 \delta+2) b d f h]\right)\right)}{\left(\left(d f^{-1+n} \prod_{\delta=0}^{-2+n}[1-(2 \delta+1) b d h l]\right) /\left(l^{n-1} \prod_{\delta=0}^{-2+n}[1+(2 \delta+2) b d f h]\right)\right)} \\
& \left(1-\left(\left(h l^{n-1} \prod_{\delta=0}^{-2+n}[1+(2 \delta+1) b d f h]\right) /\left(f^{-1+n} \prod_{\delta=0}^{-2+n}[1-(2 \delta+1) b d h l]\right)\right)\right. \\
& \left(\left(b f^{-1+n} \prod_{\delta=0}^{n-2}[1-(2 \delta) b d h l]\right) /\left(l^{n-1} \prod_{\delta=0}^{-2+n}[1+(2 \delta+1) b d f h]\right)\right) \\
& \left(\left(l^{n-1} \prod_{\delta=0}^{-2+n}[1+(2 \delta) b d f h]\right) /\left(f^{-2+n} \prod_{\delta=0}^{-2+n}[1-(2 \delta) b d h l]\right)\right) \\
& \left.\left(\left(d f^{-2+n} \prod_{\delta=0}^{n-3}[1-(2 \delta+1) b d h l]\right) /\left(l^{n-2} \prod_{\delta=0}^{n-3}[1+(2+2 \delta) b d f h]\right)\right)\right) \\
& =\frac{l^{n} \prod_{\delta=0}^{-2+n}[1+(2 \delta+2) b d f h]}{f^{-1+n}[1-(2 n-3) b d h l] \prod_{\delta=0}^{-2+n}[1-(2 \delta) b d h l](1-(b d h l /[1-(2 n-3) b d h l]))} \\
& =\frac{l^{n} \prod_{\delta=0}^{-2+n}[1+(2 \delta+2) b d f h]}{f^{-1+n} \prod_{\delta=0}^{-2+n}[1-(2 \delta) b d h l]([1-(2 n-3) b d h l]-b d h l)} \\
& =\frac{l^{n} \prod_{\delta=0}^{-2+n}[1+(2+2 \delta) b d f h]}{f^{-1+n} \prod_{\delta=0}^{-2+n}[1-(2 \delta) b d h l][1-(2 n-2) b d h l]} \\
& =\frac{l^{n} \prod_{\delta=0}^{-1+n}[1+(2 \delta) b d f h]}{f^{-1+n} \prod_{\delta=0}^{-1+n}[1-(2 \delta) b d h l]} .
\end{aligned}
$$


Obviously, the subsequent unassertive relations follows, and this complete the proof.

\section{On $3^{\text {rd }}$ System}

$$
\begin{aligned}
& x_{n+1}=\frac{x_{n-3} y_{n-4}}{y_{n}\left(1+x_{n-1} y_{n-2} x_{n-3} y_{n-4}\right)}, \\
& y_{n+1}=\frac{y_{n-3} x_{n-4}}{x_{n}\left(-1-y_{n-1} x_{n-2} y_{n-3} x_{n-4}\right)} .
\end{aligned}
$$

In this section, we will explore the solutions of following rational systems:

$$
\begin{aligned}
& x_{n+1}=\frac{x_{n-3} y_{n-4}}{y_{n}\left(1+x_{n-1} y_{n-2} x_{n-3} y_{n-4}\right)}, \\
& y_{n+1}=\frac{y_{n-3} x_{n-4}}{x_{n}\left(-1-y_{n-1} x_{n-2} y_{n-3} x_{n-4}\right)} .
\end{aligned}
$$

Theorem 3. Let $\left\{x_{n}, y_{n}\right\}$ be a solution of (20), and also let $x_{-\delta}, y_{-\delta}(\delta=4,3, \ldots, 0)$, respectively, are $a, b, c, d, e, f, g, h$, $k, l$. Then one has

$$
\begin{aligned}
& x_{4 n-4}=\frac{e^{n}}{a^{n-1} \prod_{\delta=0}^{-1+n}((1+(2 \delta)) c e g k)}, \\
& x_{4 n-3}=\frac{b \times f^{n}}{l^{n} \prod_{\delta=0}^{n-1}[1+(1+2 \delta) b d f h]}, \\
& x_{4 n-2}=\frac{c(-e)^{n}(a c g k+1)^{n}}{a^{n} \prod_{\delta=0}^{n-1}[1+(1+2 \delta) c e g k]}, \\
& x_{4 n-1}=\frac{d(-f)^{n}(1+b d h l)^{n}}{l^{n} \prod_{\delta=0}^{-1+n}[1+(2+2 \delta) b d f h]}, \\
& y_{4 n-4}=\frac{l^{n} \prod_{\delta=0}^{-1+n}[1+(2 \delta) b d f h]}{f^{-1+n}}, \\
& y_{4 n-3}=\frac{g(-a)^{n} \prod_{\delta=0}^{-1+n}[1+(2 \delta) c e g k]}{e^{n}(1+a c g k)^{n}}, \\
& y_{4 n-2}=\frac{h(-l)^{n} \prod_{\delta=0}^{-1+n}[1+(2 \delta+1) b d f h]}{f^{n}(1+b d h l)^{n}}, \\
& y_{4 n-1}=\frac{k a^{n} \prod_{\delta=0}^{-1+n}[1+(2 \delta+1) c e g k]}{e^{n}} .
\end{aligned}
$$

Proof. Obviously results true if $n=0$. Assuming that for $n-$ 1 and $n-2$ results hold, that is

$$
\begin{aligned}
& x_{4 n-9}=\frac{d(-f)^{n-2}(1+b d h l)^{n-2}}{l^{n-2} \prod_{\delta=0}^{n-3}[1+(2 \delta+2) b d f h]}, \\
& x_{4 n-8}=\frac{e^{n-1}}{a^{n-2} \prod_{\delta=0}^{-2+n}[(1+(2 \delta)) c e g k]}, \\
& x_{4 n-7}=\frac{b \times f^{-1+n}}{l^{n-1} \prod_{\delta=0}^{n-2}[1+(2 \delta+1) b d f h]}, \\
& x_{4 n-6}=\frac{c(-e)^{n-1}(1+a c g k)^{n-1}}{a^{n-1} \prod_{\delta=0}^{-2+n}[(2 \delta+1) c e g k+1]}, \\
& x_{4 n-5}=\frac{d(-f)^{n-1}(1+b d h l)^{n-1}}{l^{n-1} \prod_{\delta=0}^{-2+n}[(2+2 \delta) b d f h+1]}, \\
& y_{4 n-9}=\frac{k a^{n-2} \prod_{\delta=0}^{n-3}[1+(1+2 \delta) c e g k]}{e^{n-2}}, \\
& y_{4 n-8}=\frac{l^{n-1} \prod_{\delta=0}^{-2+n}[1+(2 \delta) b d f h]}{f^{-2+n}}, \\
& y_{4 n-7}=\frac{g(-a)^{n-1} \prod_{\delta=0}^{-2+n}[1+(2 \delta) c e g k]}{e^{n-1}(1+a c g k)^{n-1}}, \\
& y_{4 n-6}=\frac{h(-l)^{n-1} \prod_{\delta=0}^{-2+n}[1+(2 \delta+1) b d f h]}{f^{-n+1}(1+b d h l)^{n-1}}, \\
& y_{4 n-5}=\frac{k a^{n-1} \prod_{\delta=0}^{-2+n}[1+(2 \delta+1) c e g k]}{e^{n-1}} .
\end{aligned}
$$

Next, it can be seen from system (20) that 


$$
\begin{aligned}
& x_{4 n-4}=\frac{x_{4 n-8} y_{4 n-9}}{y_{4 n-5}\left(1+x_{4 n-6} y_{4 n-7} x_{4 n-8} y_{4 n-9}\right)} \\
& =\frac{1 e^{n-1} a^{n-2} \prod_{\delta=0}^{-2+n}[(1+(2 \delta)) \operatorname{ceg} k] / k a^{n-2} \prod_{\delta=0}^{n-3}[1+(2 \delta+1) c e g k] e^{n-2}}{\left(\left(k a^{n-1} \prod_{\delta=0}^{n-2}[1+(1+2 \delta) \operatorname{ceg} k]\right) /\left(e^{n-1}\right)\right)\left(1+\left(\left(c(-e)^{n-1}(1+a c g k)^{n-1}\right) /\left(a^{n-1} \prod_{\delta=0}^{n-2}[1+(1+2 \delta) c e g k]\right)\right)\right.} \\
& \left(\left(g(-a)^{n-1} \prod_{\delta=0}^{-2+n}[1+(2 \delta) \operatorname{ceg} k]\right) /\left(e^{n-1}(1+a c g k)^{n-1}\right)\right) \\
& \left(\left(e^{n-1}\right) /\left(a^{n-2} \prod_{\delta=0}^{-2+n}[(1+(2 \delta)) c e g k]\right)\right) \\
& \left.\left(\left(k a^{n-2} \prod_{\delta=0}^{n-3}[1+(2 \delta+1) \operatorname{ceg} k]\right) /\left(e^{n-2}\right)\right)\right) \\
& =\frac{e^{n} \prod_{\delta=0}^{n-3}[1+(2 \delta+1) \operatorname{ceg} k]}{a^{n-1} \prod_{\delta=0}^{-2+n}[(1+(2 \delta)) \operatorname{ceg} k] \prod_{\delta=0}^{-2+n}[1+(2 \delta+1) \operatorname{ceg} k]\left(1+\left(\left(\operatorname{ceg} k \prod_{\delta=0}^{n-3}[1+(1+2 \delta) \operatorname{ceg} k]\right) /\left(\prod_{\delta=0}^{n-2}[1+(1+2 \delta) \operatorname{ceg} k]\right)\right)\right)} \\
& =\frac{e^{n}}{a^{n-1} \prod_{\delta=0}^{-2+n}[(1+(2 \delta)) \operatorname{ceg} k][1+(2 n-3) \operatorname{ceg} k](1+(\operatorname{ceg} k /[1+(2 n-3) \operatorname{ceg} k]))} \\
& =\frac{e^{n}}{\left.a^{n-1} \prod_{\delta=0}^{-2+n}(1+(2 \delta)) c e g k\right)([1+(2 n-3) c e g k]+c e g k)} \\
& =\frac{e^{n}}{a^{n-1} \prod_{\delta=0}^{-2+n}[(1+(2 \delta)) c e g k](1+(2 n-2) c e g k)} \\
& =\frac{e^{n}}{a^{n-1} \prod_{\delta=0}^{-1+n}[(2 \delta+1) \operatorname{ceg} k]}, \\
& y_{4 n-4}=\frac{y_{4 n-8} x_{4 n-9}}{x_{4 n-5}\left(-1-y_{4 n-6} x_{4 n-7} y_{4 n-8} x_{4 n-9}\right)} \\
& =\frac{\left(\left(l^{n-1} \prod_{\delta=0}^{-2+n}[1+(2 \delta) b d f h]\right) /\left(f^{-2+n}\right)\right)\left(\left(d(-f)^{n-2}(1+b d h l)^{n-2}\right) /\left(l^{n-2} \prod_{\delta=0}^{n-3}[1+(2 \delta+2) b d f h]\right)\right)}{\left(\left(d(-f)^{n-1}(1+b d h l)^{n-1}\right) /\left(l^{n-1} \prod_{\delta=0}^{-2+n}[1+(2 \delta+2) b d f h]\right)\right)} \\
& \left(-1-\left(\left(h(-l)^{n-1} \prod_{\delta=0}^{-2+n}[1+(2 \delta+1) b d f h]\right) /\left(f^{-1+n}(1+b d h l)^{n-1}\right)\right)\right. \\
& \left(\left(b f^{-1+n}\right) /\left(l^{n-1} \prod_{\delta=0}^{-2+n}[1+(2 \delta+1) b d f h]\right)\right) \\
& \left(\left(l^{n-1} \prod_{\delta=0}^{-2+n}[1+(2 \delta) b d f h]\right) /\left(f^{-2+n}\right)\right) \\
& \left.\left(\left(d(-f)^{n-2}(1+b d h l)^{n-2}\right) /\left(l^{n-2} \prod_{\delta=0}^{n-3}[1+(2 \delta+2) b d f h]\right)\right)\right) \\
& =\frac{(-1)^{n-2} l^{n} \prod_{\delta=0}^{-2+n}[1+(2 \delta+2) b d f h]}{(-f)^{n-1}(1+b d h l)(-1+(b d h l /(1+b d h l)))} \\
& =\frac{-l^{n} \prod_{\delta=0}^{-2+n}[1+(2 \delta+2) b d f h]}{f^{-1+n}(1+b d h l)(-1+(b d h l /(1+b d h l)))} \\
& =\frac{-l^{n} \prod_{\delta=0}^{-1+n}[1+(2 \delta) b d f h]}{f^{-1+n}(-1-b d h l+b d h l)} \\
& =\frac{l^{n} \prod_{\delta=0}^{-1+n}[1+(2 \delta) b d f h]}{f^{-1+n}} .
\end{aligned}
$$


Now, hereafter, we prove an extra result. It is noted that system (20) leads to

$$
\begin{aligned}
& x_{4 n-3}=\frac{x_{4 n-7} y_{4 n-8}}{y_{4 n-4}\left(1+x_{4 n-5} y_{4 n-6} x_{4 n-7} y_{4 n-8}\right)} \\
& =\frac{\left(\left(b f^{-1+n}\right) /\left(l^{n-1} \prod_{\delta=0}^{-2+n}[1+(2 \delta+1) b d f h]\right)\right)\left(\left(l^{n-1} \prod_{\delta=0}^{n-2}[1+(2 \delta) b d f h]\right) /\left(f^{-2+n}\right)\right)}{\left(\left(l^{n} \prod_{\delta=0}^{-1+n}[1+(2 \delta) b d f h]\right) /\left(f^{-1+n}\right)\right)\left(1+\left(\left(d(-f)^{n-1}(1+b d h l)^{n-1}\right) /\left(l^{n-1} \prod_{\delta=0}^{n-2}[1+(2 \delta+2) b d f h]\right)\right)\right.} \\
& \left(\left(h(-l)^{n-1} \prod_{\delta=0}^{-2+n}[1+(2 \delta+1) b d f h]\right) /\left(f^{-1+n}(1+b d h l)^{n-1}\right)\right) \\
& \left(\left(b f^{-1+n}\right) /\left(l^{n-1} \prod_{\delta=0}^{-2+n}[1+(2 \delta+1) b d f h]\right)\right) \\
& \left.\left(\left(l^{n-1} \prod_{\delta=0}^{n-2}[1+(2 \delta) b d f h]\right) /\left(f^{-2+n}\right)\right)\right) \\
& =\frac{\left(\left(b f^{n}\right) /\left(\prod_{\delta=0}^{-2+n}[1+(2 \delta+1) b d f h]\right)\right)}{\left(\left(l^{n} \prod_{\delta=0}^{n-1}[1+(2 \delta) b d f h]\right) /\left(\prod_{\delta=0}^{n-2}[1+(2 \delta) b d f h]\right)\right)\left(1+\left(b d f h \prod_{\delta=0}^{n-2}[1+(2 \delta) b d f h] /\left(\prod_{\delta=0}^{-2+n}[1+(2+2 \delta) b d f h]\right)\right)\right)} \\
& =\frac{\left(\left(b f^{n}\right) /\left(\prod_{\delta=0}^{-2+n}[1+(2 \delta+1) b d f h]\right)\right)}{l^{n}[1+(2 n-2) b d f h](1+(b d f h /[1+(2 n-2) b d f h]))} \\
& =\frac{b f^{n}}{l^{n} \prod_{\delta=0}^{-2+n}[1+(2 \delta+1) b d f h]([1+(2 n-2) b d f h]+b d f h)} \\
& =\frac{b f^{n}}{l^{n} \prod_{\delta=0}^{-2+n}[1+(2 \delta+1) b d f h](1+(2 n-1) b d f h)} \\
& =\frac{b f^{n}}{l^{n} \prod_{\delta=0}^{-1+n}[1+(2 \delta+1) b d f h]}, \\
& y_{4 n-3}=\frac{y_{4 n-7} x_{4 n-8}}{x_{4 n-4}\left(-1-y_{4 n-5} x_{4 n-6} y_{4 n-7} x_{4 n-8}\right)} \\
& =\frac{\left(\left(g(-a)^{n-1} \prod_{\delta=0}^{-2+n}[1+(2 \delta) \operatorname{ceg} k]\right) /\left(e^{n-1}(1+a c g k)^{n-1}\right)\right)\left(\left(e^{n-1}\right) /\left(a^{n-2} \prod_{\delta=0}^{-2+n}[(1+(2 \delta)) \operatorname{ceg} k]\right)\right)}{\left(\left(e^{n}\right) /\left(a^{n-1} \prod_{\delta=0}^{-1+n}[(1+(2 \delta)) \operatorname{ceg} k]\right)\right)} \\
& \left(-1-\left(\left(k a^{n-1} \prod_{\delta=0}^{-2+n}[1+(2 \delta+1) c e g k]\right) /\left(e^{n-1}\right)\right)\right. \\
& \left(\left(c(-e)^{n-1}(1+a c g k)^{n-1}\right) /\left(a^{n-1} \prod_{\delta=0}^{-2+n}[1+(2 \delta+1) c e g k]\right)\right) \\
& \left(\left(g(-a)^{n-1} \prod_{\delta=0}^{-2+n}[1+(2 \delta) \operatorname{ceg} k]\right) /\left(e^{n-1}(1+a c g k)^{n-1}\right)\right) \\
& \left.\left(\left(e^{n-1}\right) /\left(a^{n-2} \prod_{\delta=0}^{-2+n}[(1+(2 \delta)) c e g k]\right)\right)\right) \\
& =\frac{\left(\left(g a(-1)^{n-1}\right) /\left((1+a c g k)^{n-1}\right)\right)}{\mid e^{n} a^{n-1} \prod_{\delta=0}^{-1+n}[(1+(2 \delta)) \operatorname{ceg} k](-1-a c g k)} \\
& =\frac{g(-1)^{n-1} a^{n} \prod_{\delta=0}^{-1+n}[(1+(2 \delta)) \operatorname{ceg} k]}{e^{n}(1+a c g k)^{n-1}(-1-a c g k)} \\
& =\frac{g(-a)^{n} \prod_{\delta=0}^{-1+n}[1+2 \delta c e g k]}{e^{n}(1+a c g k)^{n}} .
\end{aligned}
$$


In a similar way, one can establish other formulas.

\section{On $4^{\text {th }}$ System}

$$
\begin{aligned}
& x_{n+1}=\frac{x_{n-1} y_{n-3}}{y_{n-1}\left(1+x_{n-1} y_{n-3}\right)}, \\
& y_{n+1}=\frac{y_{n-1} x_{n-3}}{x_{n-1}\left(-1+y_{n-1} x_{n-3}\right)} .
\end{aligned}
$$

This section determines and studies the formulas for solutions of the following nonlinear system:

$$
\begin{aligned}
& x_{n+1}=\frac{x_{n-3} y_{n-4}}{y_{n}\left(1+x_{n-1} y_{n-2} x_{n-3} y_{n-4}\right)}, \\
& y_{n+1}=\frac{y_{n-3} x_{n-4}}{x_{n}\left(-1+y_{n-1} x_{n-2} y_{n-3} x_{n-4}\right)} .
\end{aligned}
$$

Theorem 4. Let $\left\{x_{n}, y_{n}\right\}$ be a solution to (26), and also let $x_{-\delta}, y_{-\delta}(\delta=4,3, \ldots, 0)$, respectively, are $a, b, c, d, e, f, g, h$, $k, l$. Then one has

$$
\begin{aligned}
& x_{4 n-4}=\frac{e^{n}}{a^{n-1} \prod_{\delta=0}^{-1+n}[1+(2 \delta) c e g k]}, \\
& x_{4 n-3}=\frac{b \times f^{n}}{l^{n} \prod_{\delta=0}^{n-1}[1+(2 \delta+1) b d f h]}, \\
& x_{4 n-2}=\frac{c e^{n}[-1+a c g k]^{n}}{a^{n} \prod_{\delta=0}^{n-1}[1+(2 \delta+1) c e g k]}, \\
& x_{4 n-1}=\frac{d f^{n}[-1+b d h l]^{n}}{l^{n} \prod_{\delta=0}^{-1+n}[1+(2 \delta+2) b d f h]}, \\
& y_{4 n-4}=\frac{l^{n} \prod_{\delta=0}^{-1+n}[1+(2 \delta) b d f h]}{f^{-1+n}}, \\
& y_{4 n-3}=\frac{g a^{n} \prod_{\delta=0}^{n-1}[1+(2 \delta) c e g k]}{e^{n}(-1+a c g k)^{n}}, \\
& y_{4 n-2}=\frac{h l^{n} \prod_{\delta=0}^{-1+n}[1+(2 \delta+1) b d f h]}{f^{n}(-1+b d h l)^{n}}, \\
& y_{4 n-1}=\frac{k a^{n} \prod_{\delta=0}^{-1+n}[1+(2 \delta+1) c e g k]}{e^{n}} .
\end{aligned}
$$

Proof. Obviously results true if $n=0$. Assuming that for $n-$ 1 and $n-2$ results hold, that is

$$
\begin{aligned}
& x_{4 n-9}=\frac{d f^{-2+n}[-1+b d h l]^{n-2}}{l^{n-2} \prod_{\delta=0}^{n-3}[1+(2 \delta+2) b d f h]}, \\
& x_{4 n-8}=\frac{e^{n-1}}{a^{n-2} \prod_{\delta=0}^{-2+n}[1+(2 \delta) c e g k]},
\end{aligned}
$$

$$
\begin{aligned}
& x_{4 n-7}=\frac{b f^{-1+n}}{l^{n-1} \prod_{\delta=0}^{n-2}[1+(2 \delta+1) b d f h]}, \\
& x_{4 n-6}=\frac{c e^{n-1}[-1+a c g k]^{n-1}}{a^{n-1} \prod_{\delta=0}^{-2+n}[1+(2 \delta+1) c e g k]},
\end{aligned}
$$$$
x_{4 n-5}=\frac{d f^{-1+n}[-1+b d h l]^{n-1}}{l^{n-1} \prod_{\delta=0}^{-2+n}[1+(2 \delta+2) b d f h]},
$$$$
y_{4 n-9}=\frac{k a^{n-2} \prod_{\delta=0}^{n-3}[1+(2 \delta+1) c e g k]}{e^{n-2}},
$$$$
y_{4 n-8}=\frac{l^{n-1} \prod_{\delta=0}^{-2+n}[1+(2 \delta) b d f h]}{f^{-2+n}},
$$$$
y_{4 n-7}=\frac{g a^{n-1} \prod_{\delta=0}^{n-2}[1+(2 \delta) c e g k]}{e^{n-1}(-1+a c g k)^{n-1}},
$$

$$
\begin{aligned}
& y_{4 n-6}=\frac{h l^{n-1} \prod_{\delta=0}^{-2+n}[1+(2 \delta+1) b d f h]}{f^{-1+n}(-1+b d h l)^{n-1}}, \\
& y_{4 n-5}=\frac{k a^{n-1} \prod_{\delta=0}^{-2+n}[1+(2 \delta+1) c e g k]}{e^{n-1}} .
\end{aligned}
$$

Next, one can obtain from system (26) that 


$$
\begin{aligned}
& x_{4 n-4}=\frac{x_{4 n-8} y_{4 n-9}}{y_{4 n-5}\left(1+x_{4 n-6} y_{4 n-7} x_{4 n-8} y_{4 n-9}\right)} \\
& =\frac{\left(\left(e^{n-1}\right) /\left(a^{n-2} \prod_{\delta=0}^{-2+n}[1+(2 \delta) c e g k]\right)\right)\left(\left(k a^{n-2} \prod_{\delta=0}^{n-3}[1+(2 \delta+1) c e g k]\right) /\left(e^{n-2}\right)\right)}{\left(\left(k a^{n-1} \prod_{\delta=0}^{n-2}[1+(2 \delta+1) c e g k]\right) /\left(e^{n-1}\right)\right)\left(1+\left(\left(c e^{n-1}[-1+a c g k]^{n-1}\right) /\left(a^{n-1} \prod_{\delta=0}^{n-2}[1+(2 \delta+1) c e g k]\right)\right)\right.} \\
& \left(\left(g a^{n-1} \prod_{\delta=0}^{-2+n}[1+(2 \delta) \operatorname{ceg} k]\right) /\left(e^{n-1}(-1+a c g k)^{n-1}\right)\right) \\
& \left.\left(\left(e^{n-1}\right) /\left(a^{n-2} \prod_{\delta=0}^{-2+n}[1+(2 \delta) \operatorname{ceg} k]\right)\right)\left(\left(k a^{n-2} \prod_{\delta=0}^{n-3}[1+(2 \delta+1) \operatorname{ceg} k]\right) /\left(e^{n-2}\right)\right)\right) \\
& =\frac{\left(\left(e^{n} \prod_{\delta=0}^{n-3}[1+(2 \delta+1) c e g k]\right) /\left(\prod_{\delta=0}^{-2+n}[1+(2 \delta) c e g k]\right)\right)}{a^{n-1} \prod_{\delta=0}^{-2+n}[1+(2 \delta+1) \operatorname{ceg} k]\left(1+\left(\left(\operatorname{ceg} k \prod_{\delta=0}^{n-3}[1+(2 \delta+1) \operatorname{ceg} k]\right) /\left(\prod_{\delta=0}^{-2+n}[1+(2 \delta+1) \operatorname{ceg} k]\right)\right)\right)} \\
& =\frac{e^{n}}{a^{n-1} \prod_{\delta=0}^{-2+n}[1+(2 \delta) \operatorname{ceg} k][1+(2 n-3) \operatorname{ceg} k](1+((\operatorname{ceg} k) /(1+(2 n-3) \operatorname{ceg} k)))} \\
& =\frac{e^{n}}{a^{n-1} \prod_{\delta=0}^{-2+n}[1+(2 \delta) \operatorname{ceg} k](1+(2 n-3) \operatorname{ceg} k+\operatorname{ceg} k)} \\
& =\frac{e^{n}}{a^{n-1} \prod_{\delta=0}^{-2+n}[1+(2 \delta) \operatorname{ceg} k](1+(2 n-2) \operatorname{ceg} k)} \\
& =\frac{e^{n}}{a^{n-1} \prod_{\delta=0}^{-1+n}[1+(2 \delta) \operatorname{ceg} k]} \\
& y_{4 n-4}=\frac{y_{4 n-8} x_{4 n-9}}{x_{4 n-5}\left(-1+y_{4 n-6} x_{4 n-7} y_{4 n-8} x_{4 n-9}\right)} \\
& =\frac{/ l^{n-1} \prod_{\delta=0}^{-2+n}[1+(2 \delta) b d f h] f^{-2+n} / d f^{-2+n}[-1+b d h l]^{n-2} l^{n-2} \prod_{\delta=0}^{n-3}[1+(2 \delta+2) b d f h]}{\left(\left(d f^{-1+n}[-1+b d h l]^{n-1}\right) /\left(l^{n-1} \prod_{\delta=0}^{-2+n}[1+(2 \delta+2) b d f h]\right)\right)} \\
& \left(-1+\left(\left(h l^{n-1} \prod_{\delta=0}^{-2+n}[1+(2 \delta+1) b d f h]\right) /\left(f^{-1+n}(-1+b d h l)^{n-1}\right)\right)\left(\left(b f^{-1+n}\right) /\left(l^{n-1} \prod_{\delta=0}^{-2+n}[1+(2 \delta+1) b d f h]\right)\right)\right. \\
& \left.\left(\left(l^{n-1} \prod_{\delta=0}^{-2+n}[1+(2 \delta) b d f h]\right) /\left(f^{-2+n}\right)\right)\left(\left(d f^{-2+n}[-1+b d h l]^{n-2}\right) /\left(l^{n-2} \prod_{\delta=0}^{n-3}[1+(2 \delta+2) b d f h]\right)\right)\right) \\
& =\frac{l^{n} \prod_{\delta=0}^{-2+n}[1+(2 \delta+2) b d f h]}{f^{-1+n}[-1+b d h l](-1+((b d h l) /(-1+b d h l)))} \\
& =\frac{l^{n} \prod_{\delta=0}^{-2+n}[1+(2 \delta+2) b d f h]}{f^{-1+n}(1-b d h l+b d h l)} \\
& =\frac{l^{n} \prod_{\delta=0}^{-1+n}[1+(2 \delta) b d f h]}{f^{-1+n}} .
\end{aligned}
$$


Also from system (26) one gets:

$$
\begin{aligned}
& x_{4 n-3}=\frac{x_{4 n-7} y_{4 n-8}}{y_{4 n-4}\left(1+x_{4 n-5} y_{4 n-6} x_{4 n-7} y_{4 n-8}\right)} \\
& =\frac{\left(\left(b f^{-1+n}\right) /\left(l^{n-1} \prod_{\delta=0}^{-2+n}[1+(2 \delta+1) b d f h]\right)\right)\left(\left(l^{n-1} \prod_{\delta=0}^{n-2}[1+(2 \delta) b d f h]\right) /\left(f^{-2+n}\right)\right)}{\left(\left(l^{n} \prod_{\delta=0}^{n-1}[1+(2 \delta) b d f h]\right) /\left(f^{-1+n}\right)\right)\left(1+\left(\left(d f^{-1+n}[-1+b d h l]^{n-1}\right) /\left(l^{n-1} \prod_{\delta=0}^{n-2}[1+(2 \delta+2) b d f h]\right)\right)\right.} \\
& \left(\left(h l^{n-1} \prod_{\delta=0}^{-2+n}[1+(2 \delta+1) b d f h]\right) /\left(f^{-1+n}(-1+b d h l)^{n-1}\right)\right) \\
& \left.\left(\left(b f^{-1+n}\right) /\left(l^{n-1} \prod_{\delta=0}^{-2+n}[1+(2 \delta+1) b d f h]\right)\right)\left(\left(l^{n-1} \prod_{\delta=0}^{n-2}[1+(2 \delta) b d f h]\right) /\left(f^{-2+n}\right)\right)\right) \\
& =\frac{\left(\left(b f^{n}\right) /\left(\prod_{\delta=0}^{-2+n}[1+(2 \delta+1) b d f h]\right)\right)}{\left(\left(l^{n} \prod_{\delta=0}^{n-1}[1+(2 \delta) b d f h]\right) /\left(\prod_{\delta=0}^{-2+n}[1+(2 \delta) b d f h]\right)\right)\left(1+\left(\left(b d f h \prod_{\delta=0}^{n-2}[1+(2 \delta) b d f h]\right) /\left(\prod_{\delta=0}^{-2+n}[1+(2 \delta+2) b d f h]\right)\right)\right)} \\
& =\frac{\left(\left(b f^{n}\right) /\left(\prod_{\delta=0}^{-2+n}[1+(2 \delta+1) b d f h]\right)\right)}{l^{n}[1+(2 n-2) b d f h](1+((b d f h) /[1+(2 n-2) b d f h]))} \\
& =\frac{b f^{n}}{l^{n}([1+(2 n-2) b d f h]+b d f h) \prod_{\delta=0}^{-2+n}[1+(2 \delta+1) b d f h]} \\
& =\frac{b f^{n}}{l^{n}[1+(2 n-1) b d f h] \prod_{\delta=0}^{n-2}[1+(2 \delta+1) b d f h]} \\
& =\frac{b f^{n}}{l^{n} \prod_{\delta=0}^{-1+n}[1+(2 \delta+1) b d f h]} \\
& y_{4 n-3}=\frac{y_{4 n-7} x_{4 n-8}}{x_{4 n-4}\left(-1+y_{4 n-5} x_{4 n-6} y_{4 n-7} x_{4 n-8}\right)} \\
& =\frac{\left(\left(g a^{n-1} \prod_{\delta=0}^{-2+n}[1+(2 \delta) \operatorname{ceg} k]\right) /\left(e^{n-1}(-1+a c g k)^{n-1}\right)\right)\left(\left(e^{n-1}\right) /\left(a^{n-2} \prod_{\delta=0}^{-2+n}[1+(2 \delta) \operatorname{cegk}]\right)\right)}{\left(\left(e^{n}\right) /\left(a^{n-1} \prod_{\delta=0}^{-1+n}[1+(2 \delta) \operatorname{ceg} k]\right)\right)\left(-1+\left(\left(k a^{n-1} \prod_{\delta=0}^{-2+n}[1+(2 \delta+1) \operatorname{ceg} k]\right) /\left(e^{n-1}\right)\right)\left(\left(c e^{n-1}[-1+a c g k]^{n-1}\right) /\left(a^{n-1} \prod_{\delta=0}^{-2+n}[1+(2 \delta+1) c e g k]\right)\right)\right.} \\
& \left.\left(\left(g a^{n-1} \prod_{\delta=0}^{-2+n}[1+(2 \delta) \operatorname{ceg} k]\right) /\left(e^{n-1}(-1+a c g k)^{n-1}\right)\right)\left(\left(e^{n-1}\right) /\left(a^{n-2} \prod_{\delta=0}^{n-2}[1+(2 \delta) c e g k]\right)\right)\right) \\
& =\frac{\left(\left(g a^{n}\right) /\left((-1+a c g k)^{n-1}\right)\right)}{\left(\left(e^{n}\right) /\left(\prod_{\delta=0}^{n-1}[1+(2 \delta) \operatorname{ceg} k]\right)\right)(-1+a c g k)} \\
& =\frac{g a^{n} \prod_{\delta=0}^{-1+n}[1+(2 \delta) c e g k]}{e^{n}(-1+a c g k)^{n-1}(-1+a c g k)} \\
& =\frac{g a^{n} \prod_{\delta=0}^{-1+n}[1+(2 \delta) c e g k]}{e^{n}(-1+a c g k)^{n}} .
\end{aligned}
$$

Subsequent results follow from the above assertion.

\section{Numerical Simulation}

In order to verify our theoretical results, we consider four interesting numerical examples by fixing suitable initial values. These simulation shows the solutions of under consideration discrete-time systems, which are depicted in (8), (14), (20), and (26).
Example 1. This example presents that the solutions of discrete-time system (8) with $x_{-\delta}, y_{-\delta}(\delta=4,3, \ldots, 0)$, respectively, are $1,0.2,0.1,0.02,2,1.5,0.3,1,0.4,4$ as shown in Figure 1.

Example 2. This example shows the behavior of system (14). The relevant plot is given in Figure 2 under the initial conditions: $\quad x_{-\delta}, y_{-\delta}(\delta=4,3, \ldots, 0)$, respectively, are $0.5,3.5,0.01,2,0.2,1.5,3,0.1,0.7,0.3$. 


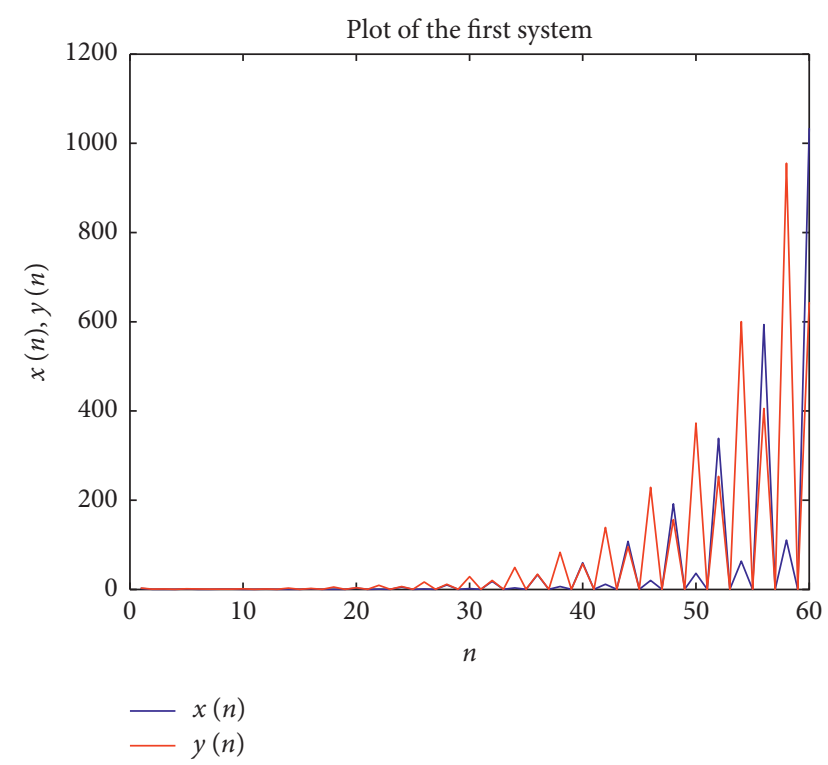

Figure 1: Plot of the solutions of $1^{\text {st }}$ system.

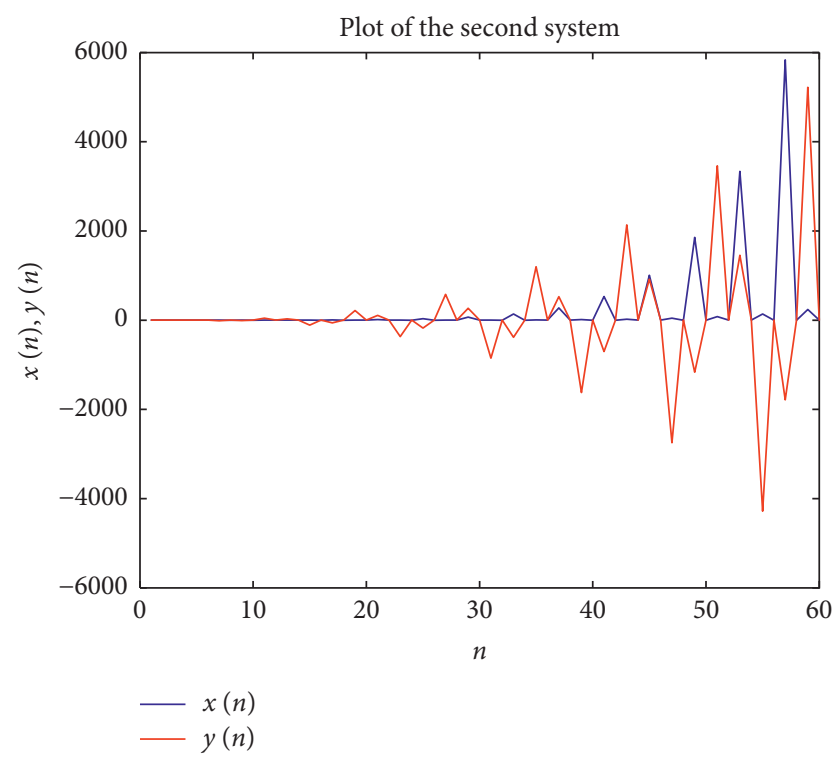

FIGURE 2: Plot of the solutions of $2^{\text {nd }}$ system.

Example 3. Figure 3 depicts the dynamics of solutions to (20) when we randomly consider the values as follows: $x_{-\delta}, y_{-\delta}(\delta=4,3, \ldots, 0), \quad$ respectively, are $0.1,0.9,0.01,0.3,0.2,0.4,0.3,0.03,0.2,0.8$.

Example 4. The behavior of system (26) are plotted in Figure 4 with $x_{-\delta}, y_{-\delta}(\delta=4,3, \ldots, 0)$, respectively, are $0.1,0.05,0.06,0.2,0.2,0.4,0.2,0.04,0.08,0.81$.

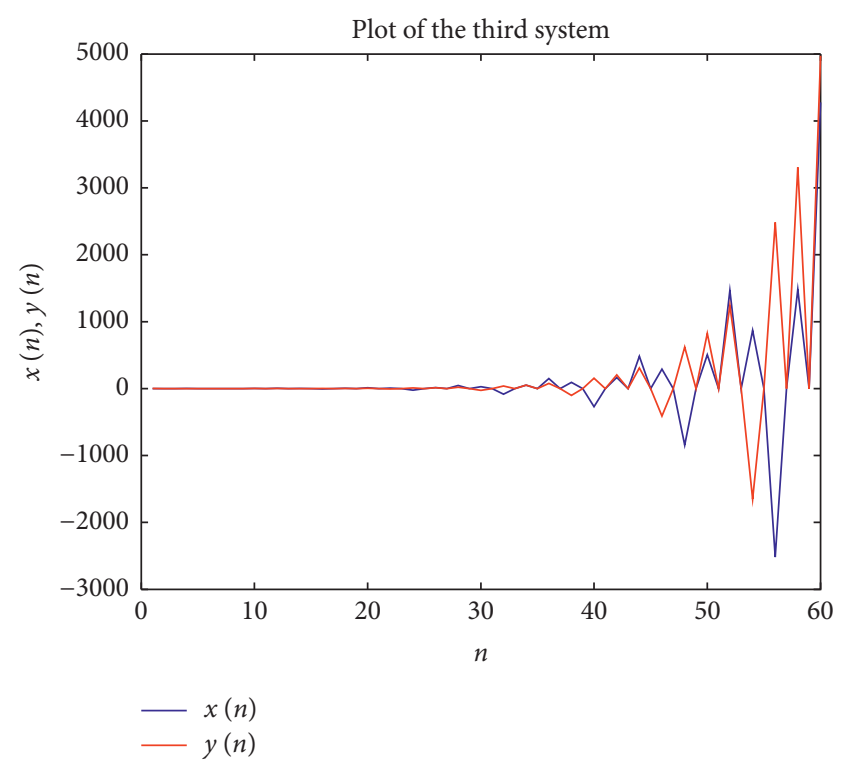

Figure 3: Plot of the solutions of $3^{\text {rd }}$ system.

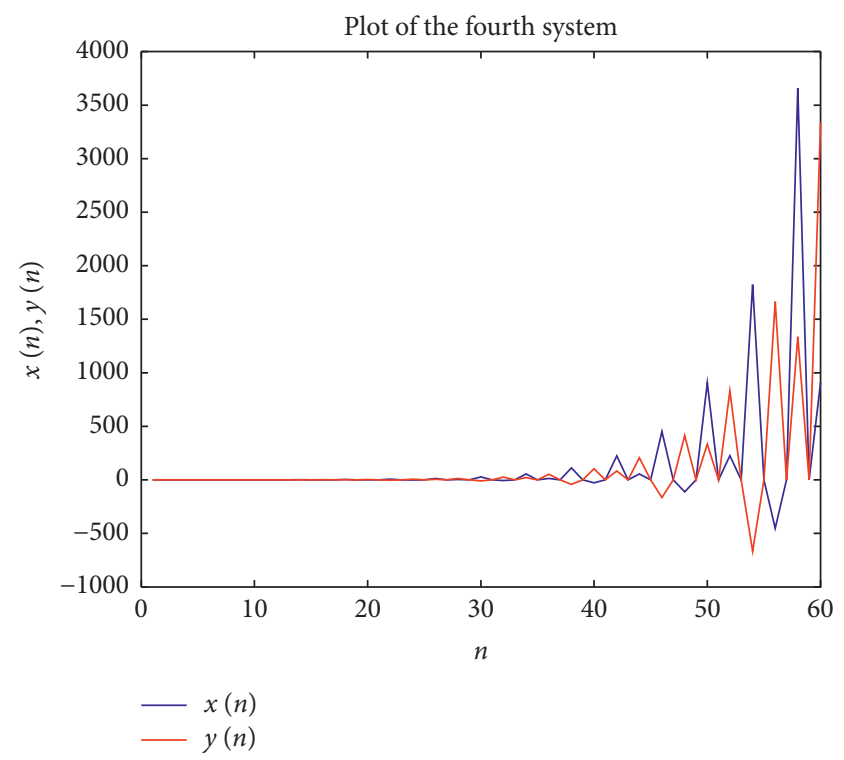

Figure 4: Plot of the solutions of $4^{\text {th }}$ system.

\section{Conclusion}

In this paper, we deal with the form of the solutions of four cases of the nonlinear systems of difference equations $x_{n+1}=$ $\left(\left(x_{n-3} y_{n-4}\right) /\left(y_{n}\left(1+x_{n-1} y_{n-2} x_{n-3} y_{n-4}\right)\right)\right), y_{n+1}=\left(\left(y_{n-3}\right.\right.$ $\left.\left.x_{n-4}\right) /\left(x_{n}\left( \pm 1 \pm y_{n-1} x_{n-2} y_{n-3} x_{n-4}\right)\right)\right)$. Finally some numerical examples are giving by fixing suitable initial values to show the qualitative behavior of under consideration systems. 


\section{Data Availability}

All the data used in this article have been included, and the sources were they where adopted were cited accordingly.

\section{Conflicts of Interest}

The authors declare that they have no conflicts of interest regarding the publication of this paper.

\section{Acknowledgments}

H. S. Alayachi and M. S. M. Noorani would like to acknowledge UKM Grant DIP-2017-011 and Ministry of Education Malaysia Grant FRGS/1/2017/STG06/UKM/01/1 for financial support while the research by A. Q. Khan and A. Khaliq was partially supported by the Higher Education Commission of Pakistan.

\section{References}

[1] A. Asiri, M. M. El-Dessoky, and E. M. Elsayed, "Solution of a third order fractional system of difference equations," Journal of Computational Analysis and Applications, vol. 24, no. 3, pp. 444-453, 2018.

[2] A. S. Kurbanli, C. Çinar, and M. E. Erdoğan, "On the behavior of solutions of the system of rational difference equations $x_{n+1}=x_{n-1} / y_{n} x_{n-1}-1, y_{n+1}=y_{n-1} /$ $x_{n} y_{n-1}-1, z_{n+1}=x_{n} / y_{n} z_{n-1}$," Applied Mathematics, vol. 2, pp. 1031-1038, 2011.

[3] H. El-Metwally and E. M. Elsayed, "Form of solutions and periodicity for systems of difference equations," Journal of Computational Analysis and Applications, vol. 15, no. 5, pp. 852-857, 2013.

[4] N. Touafek and E. M. Elsayed, "On the solutions of systems of rational difference equations," Mathematical and Computer Modelling, vol. 55, no. 7-8, pp. 1987-1997, 2012.

[5] E. M. Elsayed, "Solutions of rational difference systems of order two," Mathematical and Computer Modelling, vol. 55, no. 3-4, pp. 378-384, 2012.

[6] E. O. Alzahrani, M. M. El-Dessoky, E. M. Elsayed, and Y. Kuang, "Solutions and properties of some degenerate systems of difference equations," Journal of Computational Analysis and Applications, vol. 18, no. 2, pp. 321-333, 2015.

[7] A. Asiri and E. M. Elsayed, "Dynamics and solutions of some recursive sequences of higher order," Journal of Computational Analysis \& Applications, vol. 26, no. 1, pp. 656-670, 2019.

[8] A. Khaliq, H. S. Alayachi, M. S. M. Noorani, and A. Q. Khan, "On stability analysis of higher-order rational difference equation," Discrete Dynamics in Nature and Society, vol. 2020, pp. 1-10, 2020.

[9] A. Q. Khan and S. M. Qureshi, "Global dynamical properties of rational higher-order system of difference equations," Discrete Dynamics in Nature and Society, vol. 2020, pp. 1-15, 2020.

[10] M. M. El-Dessoky, "The form of solutions and periodicity for some systems of third-order rational difference equations," Mathematical Methods in the Applied Sciences, vol. 39, no. 5, pp. 1076-1092, 2016.

[11] M. M. El-Dessoky, E. M. Elsayed, E. M. Elabbasy, and A. Asiri, "Expressions of the solutions of some systems of difference equations," Journal of Computational Analysis \& Applications, vol. 27, no. 1, pp. 1161-1172, 2019.

[12] M. M. El-Dessoky, A. Khaliq, A. Asiri, and A. Abbas, "Global attractivity and periodic nature of a higher order difference equation," Journal of Computational Analysis \& Applications, vol. 26, no. 1, pp. 294-304, 2019.

[13] M. M. El-Dessoky, M. Mansour, and E. M. Elsayed, "Solutions of some rational systems of difference equations," Utilitas Mathematica, vol. 92, pp. 329-336, 2013.

[14] E. M. Elsayed, "On the solutions of a rational system of difference equations," Fasciculi Mathematici, vol. 45, pp. 25-36, 2010.

[15] E. M. Elsayed and A. M. Ahmed, "Dynamics of a three-dimensional systems of rational difference equations," Mathematical Methods in the Applied Sciences, vol. 39, no. 5, pp. 1026-1038, 2016.

[16] A. Gurbanlyyev, "On a system of difference equations," European Journal of Mathematics and Computer Science, vol. 3, no. 1, pp. 1-14, 2016.

[17] Y. Halim, "Global character of systems of rational difference equations," Electronic Journal of Mathematical Analysis and Applications, vol. 3, no. 1, pp. 204-214, 2015.

[18] T. F. Ibrahim, "Two-dimensional fractional system of nonlinear difference equations in the modeling competitive populations," International Journal of Basic \& Applied Sciences, vol. 12, no. 5, pp. 103-121, 2012.

[19] L. Keying, Z. Zhongjian, L. Xiaorui, and L. Peng, "More on three-dimensional systems of rational difference equations," International Journal of Basic \& Applied Sciences, vol. 2011, p. 9, 2011.

[20] A. Q. Khan and M. N. Qureshi, "Global dynamics of a competitive system of rational difference equations," Mathematical Methods in the Applied Sciences, vol. 38, no. 18, pp. 4786-4796, 2015.

[21] H. S. Alayachi, M. S. M. Noorani, and E. M. Elsayed, "Qualitative analysis of a fourth order difference equation," Journal of Applied Analysis and Computation, vol. 10, no. 4, pp. 1343-1354, 2020.

[22] A. S. Kurbanli, C. Çinar, and D. Şımşek, "On the periodicity of solutions of the system of rational difference equations," Applied Mathematics, vol. 2, no. 4, pp. 410-413, 2011.

[23] O. Özkan and A. S. Kurbanli, "On a system of difference equations," Discrete Dynamics in Nature and Society, vol. 2013, p. 7, 2013.

[24] M. Phong, "A note on a system of two nonlinear difference equations," Electronic Journal of Mathematical Analysis and Applications, vol. 3, no. 1, pp. 170-179, 2015.

[25] I. Yalcinkaya, "On the global asymptotic stability of a secondorder system of difference equations," Discrete Dynamics in Nature and Society, vol. 2008, p. 12, 2008.

[26] C. Tunç and O. Tunç, "A note on certain qualitative properties of a second order linear differential system," Discrete Dynamics in Nature and Society, vol. 9, no. 2, pp. 953-956, 2015.

[27] C. Tunç and O. Tunç, "On the boundedness and integration of non-oscillatory solutions of certain linear differential equations of second order," Journal of Advanced Research, vol. 7, no. 1, pp. 165-168, 2016.

[28] C. Tunç and O. Tunç, "A note on the stability and boundedness of solutions to non-linear differential systems of second order," Journal of the Association of Arab Universities for Basic and Applied Sciences, vol. 24, no. 1, pp. 169-175, 2017. 